\title{
Epidemiology and genetics of diabetic complications
}

\author{
R .S. N ewfield ${ }^{1}$, M. Polak ${ }^{2}$, R. M archase $^{3}$, P. Czernichow ${ }^{2}$ \\ ${ }^{1}$ Cornell University Medical College, New York, New York, USA \\ ${ }^{2}$ Service d'Endocrinologie et Diabétologie Pédiatriques, Hôpital Robert Debré, Paris, France \\ ${ }^{3}$ Department of Cell Biology, University of Alabama, Birmingham, Alabama, USA
}

Although hyperglycaemia is undoubtedly the major risk factor for the development of late diabetic complications in insulin-dependent diabetes mellitus (IDDM), it is clear that other exposures and/or genetic susceptibilities play a role. While knowledge concerning the genetic determinants predisposing towards or protecting against developing IDDM is incomplete, it is well-established (e.g. HLA markers). In contrast, researchers are still in search of reliable markers that reflect a genetic susceptibility to develop specific diabetic complications, or protective genotypes. The search for such markers was the topic of three presentations given by Dr. Krolewski from the Joslin Diabetes Center, Boston, USA, by Dr. Jeunemaitre, INSERM, Paris, France and by Dr. Marre, Angers, France. We here by summarize some of the approaches and uses of epidemiological and/or family studies to assess whether a genetic susceptibility exists, and if so, how to identify such a genotype. Clear definition of the phenotype and the ethnic constitution of the study population, as well as careful documentation of glycaemic control and duration of diabetes, cannot be over-emphasized.

Andrzej Krolewski: Evidence for genetic susceptibility to diabetic complications and epidemiological aspects

Three characteristics of the occurrence of complications can be used to evaluate the possible contributions of genetic determinants: 1) magnitude of

Participants: X. Jeunemaitre, College de France, Paris, France A. Krolewski, Joslin Diabetes Center, Boston, Massachusetts, USA

M. Marre, Centre Hospitalier Universitaire, Angers, France

Corresponding author: R.S. Newfield, Cornell University Medical College, 1300 York Avenue, LC604, New York, NY 10021, USA life-time risk of the specific complication; 2) variability among ethnic subpopulations; 3) magnitude of familial aggregation. Significant data exist for retinopathy and nephropathy, whereas data are lacking for neuropathy and coronary artery disease. As reported by Krolewski et al. and similarly in other large studies (e.g. EURODIAB IDDM), non-proliferative retinopathy develops in almost all IDDM patients within the first 15-20 years. It is thus unlikely that genetic factors are involved in the onset, although they may modulate progression of non-proliferative to proliferative retinopathy, which occurs in about $75 \%$ of patients with 40 years' disease duration. Alternatively, one may try to identify a protective gene in the $25 \%$ of patients in whom there is no progression. Diabetic nephropathy, defined as persistent proteinuria, was used by Dr. Krolewski as a paradigm with which to assess the genetic susceptibility of specific diabetic complications and as a means to identify genes contributing to these susceptibilities. In sharp contrast to diabetic retinopathy, only one third of all patients with IDDM develop advanced diabetic nephropathy during their lifetime. The incidence of persistent proteinuria increases during the first 15-20 years of IDDM and gradually declines thereafter. This pattern demonstrates that only a subset of IDDM patients is likely to develop persistent proteinuria; the magnitude of this subset depends in part on the degree of glycaemic control and is expected to decrease in size in the future with improved metabolic control. Familial clustering of renal complications in families with two or more IDDM siblings was previously demonstrated in two small studies. Family ascertainment and a larger sample size (110 Caucasian families with multiple IDDM siblings) has been achieved in a recent Joslin Diabetes Center study. The cumulative incidence of advanced diabetic nephropathy after 35 years was $35 \%$ in the probands, but the risk in siblings varied according to the proband's renal status. 
The cumulative risk of persistent proteinuria in siblings of probands with nephropathy was $72 \%$, compared to only $25 \%$ in the siblings of probands without nephropathy $(p<0.001)$. This large $(47 \%)$ difference is consistent with a major gene effect that makes IDDM patients susceptible to the development of diabetic nephropathy. The recent data from the Diabetes Control and Complications Trial (DCCT), having been adjusted for diabetes duration and glycaemic control, arrived at a similar conclusion. An additional observation in the DCCT was the significant intraclass correlation for diabetic nephropathy among diabetic parents and their offspring.

Xavier Jeunemaitre, Andrzej Krolewski, Michel Marre: Examination of candidate genes contributing to the development of diabetic complications: comparison of two study designs

How will we identify the major genes? One approach is to define carefully a candidate phenotype and then search for the genetic determinants of this phenotype. Hypertension is an example. A seminal observation is that parents of siblings with diabetic nephropathy have higher blood pressures than parents of patients without nephropathy. The role of hypertension in the development of diabetic nephropathy is debated, but if left untreated, hypertension clearly hastens the progression of diabetic nephropathy. Development of essential hypertension, independent of diabetes, clearly has a genetic component. Therefore, these issues raise the question of whether genes which predispose to essential hypertension also play a role in the genetic susceptibility to diabetic nephropathy. Such candidate genes are only beginning to be tested.

One strategy is a case-control study, in which one examines the distributions of DNA sequence markers in groups of unrelated IDDM patients with and without the specific complication investigated. Another strategy, called a transmission disequilibrium test (TDT), involves examination of transmission from heterozygote parents to offspring affected with a specific complication. Dr. Krolewski recommends the use of TDT as a robust, reliable and sensitive method with its main weakness being the difficulty in obtaining DNA from both parents. Case-control studies are often less reliable due to selection bias/population stratification issues.

Xavier Jeunemaitre, Andrzej Krolewski, Michel Marre: Candidate genes for the susceptibility to diabetic nephropathy and/or hypertension: the reninangiotensin system as an attractive target

A candidate gene of interest, discussed by Dr. Jeunemaitre, is the gene coding for angiotensinogen
(AGT), the precursor of angiotensin I. Studies between one AGT gene polymorphism marker (M235T) and essential hypertension showed linkage to the severe hypertensive phenotype. However there was no linkage between that marker (M235T) and diabetic nephropathy.

In 1994, Dr. Marre described in a case-control study the insertion/deletion (I/D) polymorphism of the angiotensin converting enzyme (ACE) gene as a marker for diabetic nephropathy. He showed that patients homozygous for insertion (I/I) have lower ACE plasma levels and are at reduced risk for diabetic nephropathy. Dr. Marre noted that in IDDM patients with nephropathy who received therapy with an ACE inhibitor for 8 years the decline in glomerular filtration rate was faster in patients with a $\mathrm{D} / \mathrm{D}$ genotype compared to $\mathrm{D} / \mathrm{I}$ or $\mathrm{I} / \mathrm{I}$. However, the data from a TDT study done by Dr. Krolewski are conflicting. He showed that $64 \%$ of probands with IDDM of less than 27 years' duration who progressed to persistent proteinuria had the insertion allele. However, only $42 \%$ of unaffected parents had the insertion allele, thus implicating the insertion genotype as a marker for rapid progression. Such discrepancies mark the literature concerning the role of polymorphisms of the ACE gene, either as markers for diabetic nephropathy and/or for its progression. This may be accounted for by differences in study design and/or population, but it also stresses the importance of large family studies. Regardless, in spite of the importance of the ACE enzyme in the renin-angiotensin system, it does not appear to be a major genetic marker for diabetic nephropathy. It has also been shown that it does not confer susceptibility to hypertension. However, Dr. Marre suggested that a combined gene defect, e.g. in ACE and AGT, may predispose a subgroup of patients to diabetic nephropathy.

Michel Marre: Testing of the physiological relevance of culprit genes identified by the candidate gene approach

To verify that genotype accounts for a phenotype in diseases of enzyme deficiencies, one has to document that a specific mutation causes a reduction in enzyme activity. Similarly, once a susceptibility gene has been identified, a confirmation that it accounts for the phenotype is required. Miller et al. showed that patients with IDDM of short duration bearing the ACE I/I genotype had glomerular hyperfiltration, a sign of incipient nephropathy. Dr. Marre investigated whether the D/I genotype correlates with changes in renal haemodynamic parameters in response to hyperglycaemia. He concluded that patients homozygous for the insertion are less likely to develop glomerular hyperdynamic injury. However, these conclusions are in contrast to the study by Miller et al. It would thus 
appear that effects due to polymorphisms at such loci are less than clear. Nonetheless, confirming the pathological importance of other strongly implicated candidate genes, that are yet to be identified, may be worthy of pursuit.

\section{Andrzej Krolewski: Genome scanning approach}

If no candidate genes are assumed, then one must scan the whole human genome for chromosomal region(s) containing a susceptibility gene. These chromosomal region(s) may then be investigated further to identify a specific gene. There are a number of differences in the type of families necessary for genome scanning compared to TDT study design. Families with sib pairs, i.e. multiplex, are necessary for genome scanning, and DNA from parents, which may or may not be affected, is not necessarily a prerequisite. This contrasts with simplex families that are preferable for TDT designs, i.e. one affected sibling and two unaffected parents, with DNA available from all three. Unlike TDT study design, which is not affected by ethnic variance, the power of genome scanning is enhanced by using a more homogenous population. The analysis of discordant sibpairs requires fewer families to achieve power than the use of concordant sibpairs. Moreover, one is able to predict more safely that the gene identified is responsible for the phenotype. Analysis employing discordant sibpairs is suitable for conditions where the sibling recurrence rate is high $(>50 \%)$. Thus, discordant sibpairs are ideal for diabetic nephropathy, due to a $72 \%$ sibling recurrence rate. This clearly calls for large numbers of well-defined families in order to conduct both TDT and genome scanning family studies.

\section{Future directions and recommendations}

- Future efforts aimed at identifying susceptibility genes for late diabetic complications should preferably be based on studying a large number of simplex and/or multiplex families (as indicated by study design) with a clearly defined phenotype. This approach is more robust methodologically than case-control studies.

- Large family studies call for a collaborative effort among scientists, patients/parents, and organizations (e.g. JDFI) to recruit sufficient families and funds.

- The above strategies allow the best chances for identifying susceptibility genes for late diabetic complications. Being able to define subsets of patients at high risk genetically, for developing specific diabetic complications, is extremely important, and may have critical therapeutic implications. For example, it will be useful to identify the subset of microalbuminuric patients that has the genetic susceptibility to progress to persistent proteinuria. 